\title{
From noncommutative space-time to quantum relativistic symmetries with fundamental mass parameter
}

\author{
Jerzy Lukierski \\ Institute for Theoretical Physics, University of Wrocław, \\ pl. Maxa Borna 9, 50-204 Wrocław, Poland \\ E-mail: lukier@ift.uni.wroc.pl
}

December 29, 2001

\begin{abstract}
We consider the simplest class of Lie-algebraic deformations of spacetime algebra, with the selection of $\kappa$-deformations as providing quantum deformation of relativistic framework. We recall that the $\kappa$-deformation along any direction in Minkowski space can be obtained. Some problems of the formalism of $\kappa$-deformations will be considered. We shall comment on the conformal extension of light-like $\kappa$-deformation as well as on the applications to astrophysical problems.
\end{abstract}

\section{Introduction}

Recently the noncommutative modification of space-time geometry gained remarkable attention mainly due to two reasons:

i) It has been argued (see e.g [1, 2, 3, 国) that the quantum gravity corrections can be algebraically described by the noncommutative modification of Minkowski space-time geometry

$$
\left[x_{\mu}, x_{\nu}\right]=0 \quad \longrightarrow \quad\left[\hat{x}_{\mu}, \hat{x}_{\nu}\right]=i \frac{1}{\kappa} \theta_{\mu \nu}\left(\frac{\hat{x}}{\kappa}\right)
$$

where $\kappa$ is fundamental mass-like parameter and in the general case

$$
\hat{\theta}_{\mu \nu}(\hat{x})=\theta_{\mu \nu}^{(0)}+\theta_{\mu \nu}^{(1) \lambda} \hat{x}_{\lambda}+\theta_{\mu \nu}^{(2) \lambda \tau} \hat{x}_{\lambda} \hat{x}_{\tau}+\ldots
$$

ii) The noncommutativity of space-time coordinates can be obtained also from the quantization of open string in $D=10$ in the presence of nonvanishing 2-tensor background field $B_{\mu \nu}(x)$ (see e.g. [5, 6, 7). If $B_{\mu \nu}$ is 
constant, it implies also constant value of $\theta_{\mu \nu}$. In general case the relation (11) should be treated as a part of deformed relativistic phase space algebra with generators $\left(\hat{x}_{\mu}, \hat{p}_{\mu}\right)$ and local function $\theta_{\mu \nu}(\hat{x})$ extended to the functional $\theta_{\mu \nu}[\hat{x}, \hat{p}]$ of eight phase space generators.

The most popular case of deformation (11) is provided by constant value of the commutator $\left(\theta_{\mu \nu}(\hat{x})=\theta_{\mu \nu}^{(0)}\right)$. Such assumption is also made in original Doplicher-Fredenhagen-Roberts (DFR) model [1]. In such a case the relativistic symmetries remain classical, however the presence of constant two-tensor $\theta_{\mu \nu}^{(0)}$ implies the breaking of classical Lorentz symmetry円. In local QFT models on deformed Minkowski space with constant value of $\theta_{\mu \nu}$ one obtains the standard relativistic action supplemented with Lorentz symmetry breaking terms, proportional to inverse powers of $\kappa$.

The next case is described by commutator (1) with linear term in $\hat{x}_{\mu}$.

$$
\left[\hat{x}_{\mu}, \hat{x}_{\nu}\right]=i \frac{1}{\kappa} \theta_{\mu \nu}^{(1) \rho} \hat{x}_{\rho} .
$$

This is the main subject of our considerations. In order to obtain the translational invariance of the theory

$$
\hat{x}_{\mu} \rightarrow \hat{x}_{\mu}+\hat{v}_{\mu}
$$

the basic algebra (3) imply that

$$
\left[\hat{v}_{\mu}, \hat{v}_{\nu}\right]=i \frac{1}{\kappa} \theta_{\mu \nu}^{(1) \rho} \hat{v}_{\rho}
$$

and

$$
\left[\hat{x}_{\mu}, \hat{v}_{\nu}\right]=0 .
$$

The relations (5)-(6) imply that the addition formula (4) can be described as

$$
\Delta\left(\hat{x}_{\mu}\right)=\hat{x}_{\mu} \otimes 1+1 \otimes x_{\mu}
$$

where $\hat{x}_{\mu}=\hat{x}_{\mu} \otimes 1, \hat{v}_{\mu}=1 \otimes \hat{x}_{\mu}$. We see that the relations (3)-(7) describe the Hopf algebra of noncommutative translations, with primitive coproduct (7).

An interesting question is to ask whether the noncommutative relations (3) can be extended do $D=4$ quantum Poincaré group, with generators $\left(\hat{x}_{\mu}, \hat{\Lambda}_{\mu}^{\nu}\right)$ endowed with classical coproduct

$$
\begin{aligned}
\Delta\left(\hat{\Lambda}_{\mu}{ }^{\rho}\right) & =\hat{\Lambda}_{\mu}{ }^{\nu} \hat{\otimes} \Lambda_{\nu}{ }^{\rho} \\
\Delta\left(\hat{x}_{\mu}\right) & =\hat{x}_{\rho} \otimes \Lambda^{\rho}{ }_{\mu}+1 \otimes x_{\mu} .
\end{aligned}
$$

The general answer to this question is contained in the paper by Podleś and Woronowicz [8]. In the case of Lie-algebraic deformation (3) it appears 19, 10, 11]

\footnotetext{
${ }^{1}$ It should be mentioned that one can avoid Lorentz symmetry breaking in quantum theory, by assuming that $\theta_{\mu \nu}^{(0)}$ is a constant tensor operator transforming as relativistic two-tensor under unitary rotations of Lorentz group in extended Hilbert space.
} 
that the most general choice permitting to extend (3) to Poincaré quantum group depends on a four-vector $a^{\mu}$ in the following way:

$$
\theta_{\mu \nu}^{(1) \rho}=a_{\mu} \delta_{\nu}^{\rho}-a_{\nu} \delta_{\mu}{ }^{\rho} .
$$

One can consider the following three cases:

i) If $a=(1,0,0,0)$ one obtains standard $\kappa$-deformation of Minkowski space, with quantum time coordinate $\hat{t}\left(\hat{x}_{0}=c \hat{t}\right)$

$$
\left[\hat{x}_{0}, \hat{x}_{i}\right]=\frac{i}{\kappa} \hat{x}_{i}, \quad\left[\hat{x}_{i}, \hat{x}_{j}\right]=0 .
$$

ii) If $a=\frac{1}{\sqrt{2}}(1,1,0,0)$ (light-like four-vector; $\left.a_{\mu} a^{\mu}=0\right)$ one gets $\left(\hat{x}_{ \pm}=\right.$ $\left.\frac{1}{\sqrt{2}}\left(\hat{x}_{0} \pm \hat{x}_{3}\right) ; r, s=1,2\right)$

$$
\begin{aligned}
{\left[\hat{x}_{+}, \hat{x}_{-}\right] } & =\frac{i}{\kappa} \hat{x}_{-}, & {\left[\hat{x}_{+}, \hat{x}_{r}\right] } & =\frac{i}{\kappa} \hat{x}_{r} . \\
{\left[\hat{x}_{-}, \hat{x}_{r}\right] } & =0, & & {\left[\hat{x}_{r}, \hat{x}_{s}\right]=0 . }
\end{aligned}
$$

iii) If $a=(0,1,0,0)$ one obtains the tachyonic $\kappa$-deformation, with quantum third space coordinate

$$
\begin{array}{lll}
{\left[\hat{x}_{3}, \hat{x}_{0}\right]=\frac{i}{\kappa} \hat{x}_{0},} & {\left[\hat{x}_{3}, \hat{x}_{r}\right]=\frac{i}{\kappa} \hat{x}_{r},} \\
{\left[\hat{x}_{0}, \hat{x}_{r}\right]=0,} & {\left[\hat{x}_{r}, \hat{x}_{s}\right]=0 .}
\end{array}
$$

The generalized $\kappa$-deformations, described by the choice (9) of noncommutative space-time was proposed firstly in [9] (see also [10, 11, 12]).

In Sect. 2 we shall describe some properties of standard $\kappa$-deformed symmetries. We shall consider as basic characterization of these quantum symmetries the $\kappa$-deformed Poincaré algebra which is dual as a Hopf algebra (see e.g. [13]) to the $\kappa$-deformed Poincaré group. We shall notice here some new results concerning finite $\kappa$-deformed Lorentz transformations [14].

In Sect. 3 we shall discuss the light-like $\kappa$-deformation and its extension to $\kappa$-deformation od $d=4$ conformal symmetries. It appears that only the $a_{\mu^{-}}$ dependent $\kappa$-deformations with $a_{\mu} a^{\mu}=0$ are described by classical $r$-matrices satisfying the classical Yang-Baxter equation. It has been shown 15 that such $r$-matrix can be quantized by the extended Jordanian twist method [16, 17, 18].

In Sect. 4 we discuss some recent ideas related with the astrophysical applications of $\kappa$-deformed space-time.

\section{Standard $\kappa$-deformation}

Standard $\kappa$-deformation has been obtained firstly (see [19]) by contraction of $q$-deformed anti-de-Sitter algebra. In such a formulation of the $\kappa$-deformed 
Poincaré algebra the nonrelativistic $E(3)$ algebra $\left(O(3)\right.$ generators $M_{i}+$ space translation generators $P_{i}$ ) supplemented by $P_{0}$ remains classical. We call such a basis standard. Subsequently by suitable change of basis [20] there was obtained $\kappa$-deformed Poincaré algebra in bicrossproduct basis:

a) algebraic sector $\left(M_{\mu \nu}=\left(M_{i}=\frac{1}{2} \epsilon_{i j k} M_{j k}, N_{i}=M_{i 0}\right), P_{\mu}=\left(P_{i}, P_{0}\right)\right)$

$$
\begin{aligned}
{\left[M_{\mu \nu}, M_{\rho \tau}\right] } & =i\left(\eta_{\mu \tau} M_{\nu \rho}-\eta_{\mu \rho} M_{\nu \tau}+\eta_{\nu \rho} M_{\mu \tau}-\eta_{\nu \tau} M_{\mu \rho}\right) \\
{\left[M_{i}, P_{j}\right] } & =i \epsilon_{i j k} P_{k}, \quad\left[M_{i}, P_{0}\right]=0, \\
{\left[N_{i}, P_{j}\right] } & =i \delta_{i j}\left[\frac{\kappa}{2}\left(1-e^{-\frac{2 P_{0}}{\kappa}}\right)+\frac{1}{2 \kappa} \vec{P}^{2}+\frac{1}{\kappa} P_{i} P_{j} .\right. \\
{\left[N_{i}, P_{0}\right] } & =i P_{i}, \\
{\left[P_{\mu}, P_{\nu}\right] } & =0 .
\end{aligned}
$$

b) coalgebraic sector

$$
\begin{aligned}
\Delta M_{i} & =M_{i} \otimes 1+1 \otimes M_{i}, \\
\Delta N_{i} & =N_{i} \otimes 1+e^{-\frac{P_{0}}{\kappa}} \otimes N_{i}+\frac{1}{\kappa} \epsilon_{i j k} P_{j} \otimes M_{k}, \\
\Delta P_{i} & =P_{i} \otimes 1+e^{-\frac{P_{0}}{\kappa}} \otimes P_{i}, \\
\Delta P_{0} & =P_{0} \otimes 1+1 \otimes P_{0},
\end{aligned}
$$

c) antipodes

$$
\begin{aligned}
S\left(M_{i}\right) & =-M_{i}, \\
S\left(N_{i}\right) & =-e^{\frac{P_{0}}{\kappa}} N_{i}+\frac{1}{\kappa} \epsilon_{i j k} e^{\frac{P_{0}}{\kappa}} P_{j} M_{k} \\
S\left(P_{i}\right) & =-e^{\frac{P_{0}}{\kappa}} P_{i}, \\
S\left(P_{0}\right) & =-P_{0} .
\end{aligned}
$$

One can show that the Hopf algebra (13)-(15) is described infinitesimally by the following classical $r$-matrix

$$
r_{1}=\frac{1}{\kappa}\left(N_{i} \wedge P_{i}\right)
$$

satisfying modified Yang-Baxter equation. In bicrossproduct basis we see that in comparison with classical Poincaré symmetries only the boost transformations of three-momenta are deformed. Further in bicrossproduct basis one can introduce consistently the finite Lorentz transformations of four-momentum generators by standard formulae:

$$
P_{\mu}(\vec{\beta})=e^{i \vec{\beta} \vec{N}} P_{\mu} e^{-i \vec{\beta} \vec{N}}=\sum_{n=0}^{\infty} \frac{1^{n}}{n !}\left[\vec{\beta} \vec{N},\left[\vec{\beta} \vec{N}, \ldots\left[\vec{\beta} \vec{N}, P_{\mu}\right]\right] \ldots\right]
$$


The basis relation in bicrossproduct basis which justifies the formula (16) as consistent with Hopf algebra structure is the following equality for quantum adjoint operator (see 21], chapt 2d)

$$
\operatorname{ad}_{N_{i}} f\left(P_{\mu}\right)=N_{i}^{(1)} f\left(P_{\mu}\right) S\left(N_{i}^{(2)}\right)=\left[N_{i}, f\left(P_{\mu}\right)\right]
$$

where $\Delta N_{i}=N_{i}^{(1)} \otimes N_{i}^{(2)}$ (no summation!) is given by (14). The calculation of the function $P_{\mu}(\vec{\beta})$ for the choice of boost $\vec{\beta}=(0,0, \beta)$ has been recently performed explicitly in [14].

The deformation of $D=4$ Poincaré algebra can be removed if we introduce nonlinear realization of the four-momentum operators 21, 22, 23, 2

$$
\begin{aligned}
& P_{i}^{(0)}=e^{\frac{P_{0}}{2 \kappa}} P_{i}, \\
& P_{0}^{(0)}=\kappa \sinh \frac{P_{0}}{\kappa}+\frac{1}{2 \kappa} \vec{P}^{2} .
\end{aligned}
$$

The generators $\left(M_{\mu \nu}, P_{\mu}^{(0)}\right)$ satisfy classical Poincaré algebra, with quite complicated coproduct [12]. Because the Lorentz transformations of the four-momenta $P_{\mu}^{(0)}$ are classical, it follows from the formulae $(18)-(19)$ that the finite Lorentz transformations in bicrossproduct basis can be obtained from classical Lorentz transformations by deformation map, described by the inverse formulae to the relations (19):

$$
\begin{aligned}
P_{i} & =\kappa\left(P_{0}^{(0)}+\sqrt{P_{\mu}^{(0)} P^{(0)^{\mu}}+\kappa^{2}}\right)^{-1} \cdot P_{i}^{(0)} \\
P_{0} & =\kappa \ln \left(\frac{P_{0}^{(0)}+\sqrt{P_{\mu}^{(0)} P^{(0)^{\mu}}+\kappa^{2}}}{\kappa}\right)
\end{aligned}
$$

In the classical Poincaré algebra basis $\left(M_{\mu \nu}, P_{\mu}^{(0)}\right)$ the whole effect of deformation is contained in the coproduct. In particular we stress that the coproduct of the energy operator $P_{0}^{(0)}$ is nonprimitive (nonabelian). We would like to recall that in 12] an attempt was undertaken to interpret the noncocommutative coproduct for the energy operator $P_{0}^{(0)}$ as describing geometric two-particle interactions.

\section{$3 \quad$ Light-like $\kappa$-deformation in standard basis}

The $\kappa$-deformation in standard basis leading to "quantum" light-cone direction $x_{+}=\frac{1}{\sqrt{2}}\left(x_{0}+x_{3}\right)($ see $(11-12))$ was firstly obtained in [24]. Further in [9] such a deformation was obtained in bicrossproduct basis as a special case of generalized $\kappa$-deformation, depending on arbitrary four-vector $a_{\mu}$. The equivalence of two formulations has been shown in 25] (see also [26]).

\footnotetext{
${ }^{2}$ We choose particular deformation map. The general case is discussed in [23].
} 
Let us introduce the light-cone basis $\left(a_{\mu}=\frac{1}{\sqrt{2}}(1,1,0,0), \widetilde{a}_{\mu}=\frac{1}{\sqrt{2}}(1,-1,0,0)\right.$; $\left.a_{\mu} a^{\mu}=\widetilde{a}_{\mu} \widetilde{a}^{\mu}=0, a_{\mu} \widetilde{a}^{\mu}=1 ; r=1,2\right)$

$$
\begin{aligned}
\widetilde{P}_{0} & =\widetilde{a}^{\mu} P_{\mu}=P_{0}+P_{3}, & \widetilde{P}_{3} & =a^{\mu} P_{\mu}=P_{0}-P_{3}, \quad \widetilde{P}_{r}=P_{r}, \\
\widetilde{M}_{03} & =\widetilde{a}^{\mu} a^{\nu} M_{\mu \nu}, & \widetilde{M}_{0 r} & =\widetilde{a}^{\mu} M_{\mu r}, \\
\widetilde{M}_{3 r} & =a^{\mu} M_{\mu r}, & \widetilde{M}_{12} & =M_{12} .
\end{aligned}
$$

The light-like $\kappa$-deformation of Poincaré algebra related by duality with noncommutative translation sector described by eq. (11) is given by the following Hopf algebra.

a) algebraic sector $\widetilde{M}_{i}=\frac{1}{2} \epsilon_{i j k} \widetilde{M}_{j k} ; \widetilde{N}_{i}=\widetilde{M}_{i 0}$ Besides the classical Lorentz algebra relations and classical commutativity of four-momenta we obtain

$$
\begin{aligned}
{\left[\widetilde{M}_{i}, \widetilde{P}_{0}\right]=} & 0 \\
{\left[\widetilde{M}_{i}, \widetilde{P}_{j}\right]=} & i \kappa \epsilon_{i j}\left(1-e^{-\frac{\widetilde{P}_{0}}{\kappa}}\right)+i \epsilon_{i j k} \widetilde{P}_{k} \\
{\left[\widetilde{N}_{i}, \widetilde{P}_{0}\right]=} & i \kappa \delta_{i 3}\left(1-e^{-\frac{\widetilde{P}_{0}}{\kappa}}\right)+i\left(1-\delta_{i 3}\right) \widetilde{P}_{i} \\
{\left[\widetilde{N}_{i}, \widetilde{P}_{k}\right]=} & i \delta_{i k} \widetilde{P}_{3} e^{-\frac{\widetilde{P}_{0}}{\kappa}}+i \delta_{i 3} \widetilde{P}_{k}\left(e^{-\frac{\widetilde{P}_{0}}{\kappa}}-1\right)+\frac{i}{2 \kappa} \delta_{i k}\left(\widetilde{P}_{1}^{2}+\widetilde{P}_{2}^{2}\right) \\
& -\frac{i}{\kappa}\left(1-\delta_{i 3}\right) \widetilde{P}_{i} \widetilde{P}_{k}
\end{aligned}
$$

b) coalgebraic sector and antipodes are provided by the relations (14)-(15) with the replacement $M_{i}^{\prime} \rightarrow \widetilde{M}_{i}, N_{i} \rightarrow \widetilde{N}_{i}$ and $P_{\mu} \rightarrow \widetilde{P}_{\mu}$.

The above Hopf algebra is described by the following classical $r$-matrix (see also 27)

$$
r_{2}=\frac{1}{\kappa}\left(\widetilde{N}_{i} \wedge \widetilde{P}_{i}\right)=\frac{1}{\kappa \sqrt{2}}\left[N_{3} \wedge\left(P_{0}-P_{3}\right)+\left(N_{2}-M_{1}\right) \wedge P_{2}+\left(N_{1}+M_{2}\right) \wedge P_{1}\right]
$$

which satisfies classical Yang-Baxter equation

$$
\left[\left[r_{2}, r_{2}\right]\right] \equiv\left[r_{2}^{(12)}, r_{2}^{(13)}\right]+\left[r_{2}^{(12)}, r_{2}^{(23)}\right]+\left[r_{2}^{(13)}, r_{2}^{(23)}\right]=0
$$

The classical $r$-matrix (23) can be promoted to the $r$-matrix for $D=4$ conformal group, described by $S U(4) \simeq O(4,2)$ algebra. It appears that the generators $\left(\widetilde{N}_{i}, \widetilde{P}_{i}\right)$ spanning classical $r$-matrix (23) can be described by the generators from the positive Borel subalgebra of $s l(4)$. With suitable reality conditions (see e.g. [27]) we obtain

$$
r_{2}=\frac{1}{\kappa}\left[\frac{1}{2}\left(h_{1}+h_{3}\right) \wedge e_{6}+e_{1} \wedge e_{5}-e_{3} \wedge e_{4}\right],
$$


where the root generators of $\operatorname{sl}(4)$ enter into the fundamental $4 \times 4$ matrix representation as follows (see e.g. 28])

$$
\left(\begin{array}{cccc}
h_{1} & e_{1} & e_{4} & e_{6} \\
e_{-1} & h_{2} & e_{2} & e_{5} \\
c_{-4} & e_{-2} & h_{3} & e_{3} \\
e_{-6} & e_{-5} & e_{-3} & h_{4}
\end{array}\right)
$$

Using results presented in 16, 17, 18] one can calculate the generalized twist function, providing the full Hopf algebra structure of $\kappa$-deformed $D=4$ conformal algebra [15]. In fact, we have looked also on multiparameter solution of classical YB equation, spanned by the generators belonging to Weyl algebra (Poincaré generator + dilatations). We found for the classical $r$-matrices carrying mass dimension the corresponding quantum $\kappa$-deformed conformal algebras described by suitable extended twist functions.

\section{Outlook}

Recently $\kappa$-deformations as an example of noncommutative geometry supplemented with quantum covariance group were tried as describing quantum gravity effects (see e.g. [29, 30]) and were applied to astrophysical phenomena (see e.g. 31, 32, 33]). In commutative four-momentum framework the following two ingredients of $\kappa$-deformed geometry are used:

i) The deformation of mass-shell condition?

$$
\vec{P}^{2} e^{ \pm \frac{P_{0}}{\kappa}}-\left(2 \kappa \sinh \frac{P_{0}}{\kappa}\right)^{2}=-M^{2}
$$

ii) The nonsymmetric, nonabelian composition law for the four-momenta, described by the coproduct of momentum generators (see (14)).

Both these elements of new theory lead at present to some conceptual problems. If we assume that the parameter $\kappa$ is universal (besides $\hbar$ and $c$ as the third fundamental constant) the straightforward application of formula (27) to macroscopic bodies leads to obvious contradiction with experimental results . We see that the range of applicability of the formula (27) is still not understood what is related with some new notion of elementarity in microworld physics still to be discovered. The difficulty with consistent description by $\kappa$-physics of macroworld phenomena is also connected with the appearance of nonsymmetric, nonabelian addition law for the momenta. The problem how to incorporate modified momentum conservation law into physical models remains to be solved before we can apply $\kappa$-deformed kinematics e.g. to scattering processes and description of $\kappa$-deformed Feynman diagrams [35]. At present stage there were proposed some new ideas (see e.g. [36]) but according to our estimate they are quite preliminary.

\footnotetext{
${ }^{3}$ In bicrossproduct basis one can consider two deformed mass-shell formulae related by the inversion of $\kappa: \kappa \rightarrow \kappa^{-1}$ (see [34], formula (2.34)).

${ }^{4}$ This difficulty was pointed out to the author firstly by I. Białynicki-Birula.
} 


\section{Acknowledgment}

Support by the KBN grant 5P03B05620 is acknowledged.

\section{References}

[1] S. Doplicher, K. Fredenhagen, and J.E. Roberts, Phys Lett. B 331, 39 (1994); Comm. Math. Phys. 172, 187 (1995)

[2] D.V. Ahluwalia, Phys Lett. B 33(, 301 (1994)

[3] G. Amelino-Camelia, Mod. Phys. Lett. A 9, 3415 (1994); A 11, 111 (1996)

[4] L.J. Garay, Int. Journ. Mod. Phys. 10A, 145 (1995)

[5] C.S. Chu and P.M. Ho, Nucl. Phys. B 550, 151 (1999); hep-th/9812219

[6] N. Seiberg and E. Witten, High Energy Phys. 9909, 032 (1991); hep-th/9908142

[7] R.J. Szabo, Quantum Field Theory on Noncommutative spaces; hep-th/0109162

[8] P. Podleś and S. Woronowicz, Comm. Math. Phys. 178, 61 (1996)

[9] P. Kosiński and P. Maślanka, in "From Field Theory to Quantum Groups", eds. B. Jancewicz, J.Sobczyk (World Scientific, 1996), p. 11

[10] J. Lukierski, in Proc of Alushta Conference on Recent Problems in QFT, May 1996, ed. D. Shirkov, D.I. Kazakov, and A.A. Vladimirov, Dubna 1996, p.82

[11] K. Przanowski, Czech. J. Phys. 47, 107 (1997)

[12] J. Lukierski, $\kappa$-Deformations of relativistic symmetries: recent developments, in Proc. of Quantum Group Symposium, July 1996, Goslar, ed. H.-D. Doebner and V.K. Dobrev, Heron Press, Sofia, 1997, p. 173

[13] S. Majid, Foundations of Quantum Groups, Cambridge Univ. Press, 1995

[14] N.R. Bruno, G. Amelino-Camelia, and J. Kowalski-Glikman, Deformed Boost Transformations that saturate at the Planck scale, hep-th/0107039

[15] J. Lukierski, V. Lyakhovsky, and M. Mozrzymas, in preparation

[16] P.P. Kulish, V.D. Lyakhovsky, and A.I. Mudrov, J. Math. Phys. 40, 4569 (1999); math.QA/9806014

[17] P.P. Kulish, V.D. Lyakhovsky, and M.A. del Olmo, J. Phys. A 32, 8671 (1999) 
[18] V.D. Lyakhovsky and M.A. del Olmo, nath.QA/9903065

[19] J. Lukierski, A. Nowicki, H. Ruegg, and V.N. Tolstoy, Phys Lett. B 264, $331(1991)$

[20] S. Majid and H. Ruegg, Phys Lett. B 334, 348 (1994)

[21] S. Majid, H. Ruegg, and V.N. Tolstoy, "Quantum Groups: Formalism and Applications", Proceed. of XXX Karpacz Winter School, February 1994, Polish Scientific Publishers 1994, p. 359

[22] H. Ruegg and V.N. Tolstoy, Lett. Math. Phys. 32, 85 (1995)

[23] P. Kosiński, J. Lukierski, P. Maślanka, and J. Sobczyk, Mod. Phys. Lett. 10A, 2599 (1995)

[24] A. Ballesteros, E. Herranz, M.A. del Olmo, and M. Santander, Phys Lett. B 351, 137 (1995)

[25] K. Przanowski, Acta Phys. Polon. B 28, 1635 (1997)

[26] O. Arratia, F.Herranz, and M.A. del Olmo, J. Phys. A 31, L1 (1998); q-alg/9707025

[27] J. Lukierski, P. Minnaert, and M. Mozrzymas, Phys Lett. B 371, 215 (1996)

[28] J. Lukierski, A. Nowicki, and J. Sobczyk, J. Phys. A 26, 4047 (1993)

[29] G. Amelino-Camelia, Phys Lett. B 392, 283 (1997)

[30] G. Amelino-Camelia, J. Lukierski, and A. Nowicki, Yad. Fizyka 61, 1925 (2000)

[31] R. Alonso, P. Blasi, P.L. Ghia, and A.F. Grillo, Phys. Rev. D 62, 053010 (2000)

[32] J. Kowalski-Glikman, Phys Lett. B 499, 1 (2001)

[33] G. Amelino-Camelia, J. Lukierski, and A. Nowicki, hep-th/0103227; Czech. J. Phys., in press

[34] J. Lukierski, H. Ruegg, and W. Zakrzewski, Ann. Phys. 234, 90 (1995)

[35] P. Kosiński, J. Lukierski, and P. Maślanka, Phys. Rev. D 62, 025004 (2000)

[36] G. Amelino-Camelia and M. Arzano, hep-th/0105120 